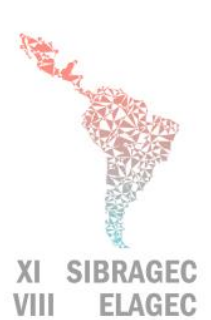

VIII ELAGEC

\section{SIMPÓSIO BRASILEIRO DE GESTÃO E ECONOMIA DA CONSTRUÇÃO}

VIII ENCUENTRO LATINOAMERICANO DE GESTIÓN Y ECONOMÍA DE LA CONSTRUCCIÓN

Do conhecimento à ação: práticas avançadas de gestão da produção Londrina, Paraná, Brasil. 23 a 25 de Outubro de 2019

\title{
CRITÉRIOS DE SUCESSO NA PERCEPÇÃO DE GESTORES DE MPE DE CONSTRUÇÃO DO SEMI ÁRIDO NORDESTINO
}

\author{
SOUSA, GLEYCIANNE C. M DE (1); COSTA, GABRIELA R. DE (2); \\ CÂNDIDO, LUÍS FELIPE (3)
}

(1) Universidade Federal do Ceará - Campus de Crateús, gleyciannecavalcante@ gmail.com (2) Universidade Federal do Ceará - Campus de Crateús, gabrielarodrigues@alu.ufc.br (3) Universidade Federal do Ceará - Campus de Crateús, luiscandido2015@gmail.com

\begin{abstract}
Despite of the importance of small business for Brazilian economy, a lack of studies in construction management literature about of this sized companies is observed. Thus, this paper aimed to analyze the perception about the success criteria of managers from Small Business of construction sector. To do so, was performed a study with managers of three construction companies from a semiarid region at northeast of Brazil. Through a qualitative and exploratory approach, 14 criteria to evaluate the organizational success were analyzed in depth comparing importance with use. It was observed that the perception of importance to each success criterion was higher than its uses. It was evidenced difficulties regarding the designers and the low importance attributed to environmental sustainability. Some similarities with medium and large business were also identified. We can conclude that the business are strictly focused on financial performance, while elementary aspects as health and safety are not achieve and reinforcing the connotation of social irresponsibility of the construction sector.
\end{abstract}

Keywords: Construction Management, Strategic Management, Continuous Improvement, Small Business.

\section{INTRODUÇÃ̃O}

A indústria da construção civil é um dos setores industriais mais importantes para o Brasil. Entretanto, o setor também é reconhecido como atrasado em relação a outros, tanto em termos de gestão como de desenvolvimento tecnológico (MELLO; AMORIM, 2009), o que tem levado a entidades (como CBIC, CNI, Fiesp, SEBRAE) e pesquisadores (BARBOSA et al. 2017; MELLO; AMORIM, 2009; MONTEIRO FILHA; COSTA; ROCHA, 2010) a realizarem diversos estudos sobre o nível de profissionalização, de produtividade, perspectivas econômicas, inovação, dentre outros, sendo poucos os estudos voltados às Micro e Pequenas Empresas (MPE) do setor.

As MPE apresentam fundamental importância econômica para o Brasil, principalmente no que se refere a geração de emprego e renda (SANTINI et al., 2015; SOUZA et al., 2014) e possuem como características marcantes a baixa profissionalização de sua gestão e a escassez de recursos financeiros (LEONE; LEONE, 2011; SOUSA, 2003), o que pode fazer com que a sua gestão seja deficitária e focada em aspectos financeiros.

Entretanto, a escassez de estudos na literatura de gestão da construção sobre MPE - que justifica o presente estudo, levou a seguinte questão de pesquisa: qual a percepção dos 
gestores de MPE sobre sucesso organizacional? Desta forma, o presente trabalho teve por objetivo analisar a percepção dos gestores de MPE quanto aos critérios de sucesso para o desempenho organizacional. Para tal, realizou-se um estudo com gestores de microempresas construtoras da cidade de Crateús-CE, semiárido nordestino.

\section{REFERENCIAL TEÓRICO}

Os Critérios de Sucesso podem ser compreendidos como uma lente para avaliar o sucesso de um empreendimento ou de uma empresa, sendo os mais conhecidos o custo, o prazo e a qualidade, chamado de triangulo de aço (TOOR; OGUNLANA, 2010). Entretanto, existe uma tendência de mudança nestes critérios na indústria da construção civil, mesmo que de forma mais lenta (CÂNDIDO; LIMA; BARROS NETO, 2016).

Neste setor, apesar das medidas que enfocam a eficiência e a redução de custos ainda predominarem - abordagem físico-financeira (CÂNDIDO; BARROS NETO; HEINECK, 2014), estudos recentes mostram mudanças significativas em nível nacional (SOUSA; CÂNDIDO; BARROS NETO, 2018) e internacional (MAYA, 2016; VILLARREAL; PELLICER; RODRIGUEZ, 2017). Porém, tais estudos têm sido realizados em médias e grandes empresas, carecendo-se de estudos em MPEs, como é o caso do presente trabalho.

\section{MÉTODO DE PESQUISA}

Esta pesquisa consistiu de um estudo qualitativo (COOPER; SCHINDLER, 2016) de caráter exploratório (COLLIS; HUSSEY, 2005) e foi realizado em três fases (Figura 1).

Figura 1 - Método de pesquisa adotado
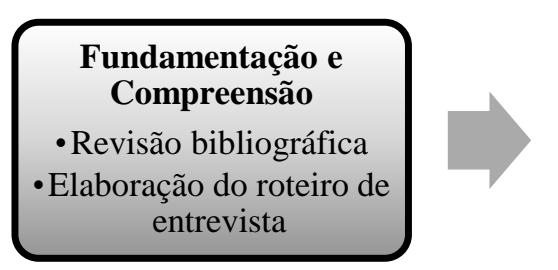

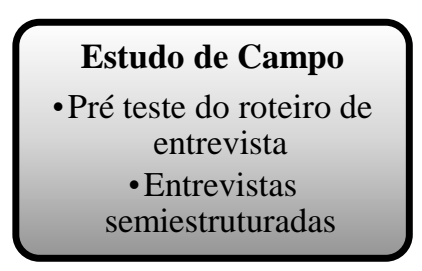

Fonte: dos autores.

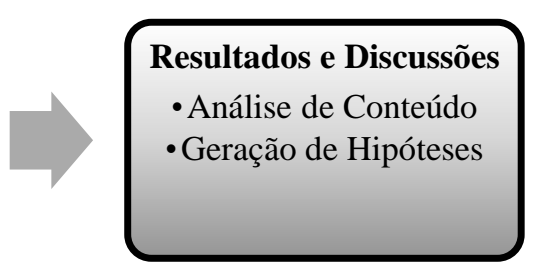

-Análise de Conteúdo

- Geração de Hipóteses

Após a primeira etapa, foram realizadas três entrevistas semiestruturadas (RICHARDSON, 2011) com gestores de MPEs construtoras de Crateús, cidade da região centro-oeste do interior do Ceará conhecida como sertão dos Inhamuns. O roteiro de entrevista continha 9 perguntas divididas entre (i) caracterização das empresas e dos respondentes -8 questões e (ii) identificação da percepção acerca dos critérios de sucesso para desempenho organizacional - 1 questão, baseada no modelo Bassioni, Price e Hassan (2005) e Bassioni, Hassan e Price (2008).

Cada respondente atribuiu um valor de 1 a 5 para a importância de cada um dos 15 critérios de sucesso e o quanto estes eram utilizados em suas empresas, sendo $1 \mathrm{sem}$ importância/sem utilização e 5 extremamente importante/extremamente utilizado. Além disso, para cada foi solicitado que os respondentes explicassem o porquê das notas.

A análise do material coletado se deu de forma qualitativa (GIBBS, 2009), através de categorias definidas a partir do modelo de modelo Bassioni, Price e Hassan (2005) e Bassioni, Hassan e Price (2008). 
SIBRAGEC - ELAGEC 2019 - de 23 a 25 de Outubro - LONDRINA - PR

\section{RESULTADOS E DISCUSSÕES}

\subsection{Caracterização das empresas e dos respondentes}

O Quadro 1 apresenta as principais características das empresas e dos respondentes, a partir do qual verifica-se similaridades entre os respondentes e as empresas que possuem o mesmo nicho de mercado, a mesma praça de atuação, o mesmo tipo de mão de obra empregada e o mesmo porte (MPEs).

Quadro 1 - Caracterização das empresas e dos respondentes

\begin{tabular}{|c|c|c|c|}
\hline Características & Empresa A & Empresa B & Empresa C \\
\hline Respondente & Proprietário & Sócio & Diretor \\
\hline Função & $\begin{array}{c}\text { Gerente } \\
\text { administrativo }\end{array}$ & $\begin{array}{l}\text { Engenheiro e } \\
\text { Administrador }\end{array}$ & Gestor \\
\hline Área de atuação & $\begin{array}{c}\text { Engenharia e } \\
\text { Administração }\end{array}$ & $\begin{array}{c}\text { Engenharia e } \\
\text { Administração }\end{array}$ & $\begin{array}{c}\text { Engenharia e } \\
\text { Administração }\end{array}$ \\
\hline Tempo de atuação & 5 anos & 22 anos & 9 anos \\
\hline Tempo de Empresa & 4,5 anos & 12 anos & 8 anos \\
\hline $\begin{array}{c}\text { Formação/Titulação } \\
\text { Máxima }\end{array}$ & $\begin{array}{l}\text { Eng. Civil - Esp. em } \\
\text { Segurança do } \\
\text { Trabaho }\end{array}$ & Engenheiro Civil & $\begin{array}{l}\text { Eng. Civil/MBA } \\
\text { Emprend. e densenv. } \\
\text { novos talentos }\end{array}$ \\
\hline Registro no Crea & Sim & Sim & Sim \\
\hline Tipo de Administração & Mista & Mista & Profissional \\
\hline Constituição da Empresa & MEI & LTDA & EIRELI \\
\hline $\begin{array}{l}\text { Quantidade de } \\
\text { Funcionários }\end{array}$ & 7 & 11 & 32 \\
\hline Praça de Atuação & Crateús & $\begin{array}{c}\text { Crateús e cidades } \\
\text { vizinhas }\end{array}$ & Crateús \\
\hline Nicho de Mercado & $\begin{array}{c}\text { Residenciais, } \\
\text { Comerciais, } \\
\text { Industriais e Públicas }\end{array}$ & $\begin{array}{l}\text { Residenciais, } \\
\text { Comerciais e } \\
\text { Públicas }\end{array}$ & $\begin{array}{l}\text { Residenciais, } \\
\text { Comerciais e } \\
\text { Públicas }\end{array}$ \\
\hline Tipo de mão de obra & $\begin{array}{l}\text { Subempreitada e } \\
\text { própria }\end{array}$ & $\begin{array}{l}\text { Subempreitada e } \\
\text { própria }\end{array}$ & $\begin{array}{c}\text { Subempreitada e } \\
\text { própria }\end{array}$ \\
\hline
\end{tabular}

Fonte: dos autores.

Verifica-se que apenas o respondente da Empresa $\mathrm{C}$ apontou que possui um tipo de administração profissional, revelando a natureza não profissional das MPEs, como observa Sousa (2003).

\subsection{Análise dos Critérios de Sucesso para o desempenho}

A Tabela 1 apresenta os resultados descritivos. Nota-se que por uma questão de viés gestores avaliando a própria empresa, verificou-se uma atribuição de altas notas para a utilização. Destarte, a nota consolidada para cada item foi obtida pela soma das notas atribuídas por cada gestor dividas pelo máximo possível. 
SIBRAGEC - ELAGEC 2019 - de 23 a 25 de Outubro - LONDRINA - PR

Tabela 1 - Percepção dos gestores quanto aos critérios de sucesso

\begin{tabular}{|c|c|c|c|c|c|c|c|c|c|c|}
\hline \multirow{2}{*}{$\begin{array}{c}\text { Perspectivas de } \\
\text { medição do } \\
\text { desempenho }\end{array}$} & \multicolumn{2}{|c|}{ A } & \multicolumn{2}{|c|}{ B } & \multicolumn{2}{|c|}{ C } & \multicolumn{2}{|c|}{ Importância } & \multicolumn{2}{|c|}{ Uso } \\
\hline & Imp. & Uso & Imp. & Uso & Imp. & Uso & Tot. & $\%$ & Tot. & $\%$ \\
\hline $\begin{array}{l}\text { A. Desempenho do } \\
\text { empreendimento/ } \\
\text { Produção/ } \\
\text { Eficiência da } \\
\text { Gestão do Projeto }\end{array}$ & 24 & 22 & 21 & 22 & 25 & 13 & 70 & 93 & 57 & 76 \\
\hline a. Custo & 5 & 5 & 4 & 5 & 5 & 5 & 14 & 93 & 15 & 100 \\
\hline b. Prazo & 5 & 5 & 5 & 5 & 5 & 2 & 15 & 100 & 12 & 80 \\
\hline $\begin{array}{l}\text { c. Qualidade e } \\
\text { Funcionalidade }\end{array}$ & 5 & 5 & 5 & 5 & 5 & 2 & 15 & 100 & 12 & 80 \\
\hline d. Produtividade & 4 & 4 & 5 & 5 & 5 & 2 & 14 & 93 & 11 & 73 \\
\hline $\begin{array}{l}\text { e. Saúde e } \\
\text { Segurança }\end{array}$ & 5 & 3 & 2 & 2 & 5 & 2 & 12 & 80 & 7 & 47 \\
\hline $\begin{array}{l}\text { B. Desempenho } \\
\text { Organizacional }\end{array}$ & 10 & 9 & 9 & 9 & 10 & 7 & 29 & 97 & 25 & 83 \\
\hline a. Financeiro & 5 & 4 & 4 & 4 & 5 & 3 & 14 & 93 & 11 & 73 \\
\hline b. Mercado & 5 & 5 & 5 & 5 & 5 & 4 & 15 & 100 & 14 & 93 \\
\hline $\begin{array}{l}\text { C. Desempenho dos } \\
\text { stakeholders } \\
\text { internos }\end{array}$ & 17 & 17 & 19 & 19 & 20 & 9 & 56 & 93 & 45 & 75 \\
\hline a. Funcionários & 4 & 4 & 5 & 5 & 5 & 2 & 14 & 93 & 11 & 73 \\
\hline $\begin{array}{l}\text { b. Parceiros } \\
\text { (subcontratados) }\end{array}$ & 4 & 4 & 5 & 5 & 5 & 1 & 14 & 93 & 10 & 67 \\
\hline c. Fornecedores & 4 & 4 & 5 & 5 & 5 & 4 & 14 & 93 & 13 & 87 \\
\hline d. Projetistas & 5 & 5 & 4 & 4 & 5 & 2 & 14 & 93 & 11 & 73 \\
\hline $\begin{array}{l}\text { D. Desempenho } \\
\text { para os stakeholders } \\
\text { externos }\end{array}$ & 18 & 18 & 18 & 17 & 17 & 10 & 53 & 53 & 45 & 75 \\
\hline $\begin{array}{l}\text { a. Impacto sobre os } \\
\text { Investidores }\end{array}$ & 5 & 5 & 3 & 3 & 3 & 3 & 11 & 73 & 11 & 73 \\
\hline $\begin{array}{l}\text { b. Impacto sobre os } \\
\text { Usuários Finais }\end{array}$ & 5 & 5 & 5 & 4 & 5 & 3 & 15 & 100 & 12 & 80 \\
\hline $\begin{array}{l}\text { c. Impacto sobre o } \\
\text { Meio Ambiente }\end{array}$ & 3 & 3 & 5 & 5 & 4 & 1 & 12 & 80 & 9 & 60 \\
\hline $\begin{array}{l}\text { d. Impacto sobre a } \\
\text { Sociedade }\end{array}$ & 5 & 5 & 5 & 5 & 5 & 3 & 15 & 100 & 13 & 87 \\
\hline
\end{tabular}

Fonte: dos autores.

Verifica-se que todos os gestores consideraram as dimensões A, B e C extremamente importantes para as suas construtoras à exceção da dimensão D. Desempenho para os stakeholders externos. Esta percepção coaduna com a literatura, pois historicamente a gestão dos stakeholders é falha na indústria da construção (KOLK; PINKSE, 2006), confirmando que há uma similaridade neste quesito entre MPE e médias e grandes empresas do setor. Verifica-se ainda a importância dada ao desempenho organizacional com ênfase em aspectos financeiros e de mercado, como também observaram Sousa, Cândido e Barros Neto (2018).

Com relação a dimensão A, verifica-se um o descaso para com a segurança e saúde, o que fica mais evidente no Quadro 3. 
SIBRAGEC - ELAGEC 2019 - de 23 a 25 de Outubro - LONDRINA - PR

\section{Quadro 3 - Percepção dos gestores quanto à Saúde e Segurança do Trabalho}

\begin{tabular}{|c|c|c|}
\hline & Ges & \\
\hline $\begin{array}{c}\text { "A dificuldade que existe é } \\
\text { a resistência ao uso de EPI } \\
\text { [...] você tem que ficar } \\
\text { exigindo insistentemente" }\end{array}$ & $\begin{array}{l}\text { “[...] a única coisa que eles } \\
\text { têm é o direito da carteira } \\
\text { assinada, mas não tem o EPI e } \\
\text { nem plano de saúde... Só o } \\
\text { que o INSS cobra por ser } \\
\text { carteira assinada" }\end{array}$ & $\begin{array}{l}\text { "Na empresa eu foco bastante na } \\
\text { questão de usar EPI, mas com } \\
\text { relação a saúde, os funcionários } \\
\text { não têm um plano, a empresa só } \\
\text { fornece auxílio/ajuda quando } \\
\text { necessário" }\end{array}$ \\
\hline
\end{tabular}

Fonte: dos autores.

Não fica evidente o porquê dos gestores darem pouca importância ao tema. No entanto, verifica-se que a principal dificuldade para este tema é a conscientização e a resistência ao uso do equipamento de proteção, ou seja, a cultura dos operários que, conforme Mello e Amorim (2009), é marcada pelo baixo grau de instrução e educação.

Em primeira análise o resultado parece contraditório, pois tipicamente as empresas cumprem no mínimo as exigências legais impostas para sua operação. Em uma segunda análise, aponta-se para a realidade das MPE e, em especial, em pequenas cidades que a municipalidade tende a não ter aparato de fiscalização efetivo, revelando a sua importância do poder público como agente de isomorfismo coercitivo (MACHADO FILHO; ZYLBERSZTAJN, 2003).

Em relação ao desempenho dos stakeholders internos os gestores alegaram algumas dificuldades, principalmente no tocante aos projetistas como evidenciado no Quadro 4.

\section{Quadro 4 - Percepção dos gestores quanto ao Desempenho stakeholders internos}

\begin{tabular}{|c|c|c|}
\hline Gestor da Empresa A & Gestor da Empresa B & Gestor da Empresa C \\
\hline "Caso particular, hoje em Crateús & O projetista pode trabalhar & "O projetista é importante, \\
podemos ver a dificuldade, pois & bem, mas a maioria atrasa & porém aqui os projetistas \\
muitos "Cadistas" vendem projetos & serviço, muitos trabalham & da cidade ainda fazem o \\
como se soubessem e as vezes até & bem, mas tem problema & básico, muitos projetos têm \\
sabem. [...] o cliente passa o & com o prazo. Meus projetos & dificuldades de serem \\
projeto, mas será que ele vai ser & quem faz são cadistas, & aprovados pois sempre \\
aprovado onde ele quer submeter?" & arquitetos, mas é difícil." & faltam alguma coisa." \\
\hline
\end{tabular}

Fonte: dos autores.

Esta realidade pode ser representativa das pequenas cidades que tendem a ter um baixo nível de profissionalização do setor construtivo. Isto pode ser reflexo de um passado recente do setor construtivo brasileiro que pouco investia em projetos.

Por fim, a dimensão D foi percebida como de menor relevância e uso. O sucesso a partir dos impactos ambientais foi um dos critérios de menor índice de utilização, pois não há fiscalização (Gestor $\mathrm{A}$ ), bem como não existem empresas que façam o manejo e disposição de RCD (Gestor C), como se observa no Quadro 5.

\section{Quadro 5 - Percepção dos gestores com relação ao Desempenho stakeholders} externos

\begin{tabular}{|c|c|c|}
\hline Gestor da Empresa A & Gestor da Empresa B & Gestor da Empresa C \\
\hline $\begin{array}{c}\text { "Se tiver fiscalização é } \\
\text { importante, se não tiver } \\
\text { não é. A realidade é } \\
\text { essa." }\end{array}$ & $\begin{array}{c}\text { "Considero importante pois } \\
\text { agora aqui em Crateús tem a } \\
\text { secretaria de meio ambiente e } \\
\text { para fazer as obras é preciso } \\
\text { licenças" }\end{array}$ & $\begin{array}{c}\text { complicada... um exemplo é o caso } \\
\text { do plano de resíduos sólidos [...] } \\
\text { aqui fica difícil, pois não existe } \\
\text { uma empresa que faz esse serviço.", }\end{array}$ \\
\hline
\end{tabular}

Fonte: dos autores. 
A baixa importância e as práticas neste tema se tornam prementes diante do grande impacto ambiental do setor (AGOPYAN; JOHN, 2011). Além disso, reforça a importância do poder público, assim como evidenciado com relação à saúde e segurança, e reforça o título de que o setor é socialmente irresponsável - como observam Chiveralls et al. (2011).

\section{CONSIDERAÇÕES FINAIS}

O presente trabalho analisou a percepção de gestores de MPE do setor da construção civil na cidade de Crateús-CE quanto aos Critérios de Sucesso para o desempenho organizacional. Verificou-se que a importância atribuída aos critérios de sucesso para desempenho não apresentou diferença significativa e em geral foi superior ao grau de utilização.

Evidenciou-se similaridades com empresas de maior porte do setor como a ênfase em aspectos financeiros e a dificuldade de gerenciamento dos stakeholders. Verificou-se, também, dificuldades com relação a segurança do trabalho e, apesar de uma alta avaliação acerca do uso da produtividade, não se evidenciou nenhum processo para o seu gerenciamento. Destaca-se ainda dificuldades com relação aos projetistas e, por fim, a baixa importância com relação a práticas ambientalmente responsáveis devido à falta de fiscalização do poder público.

Tais características constituem-se como uma contribuição deste trabalho que permite direcionar estudos mais aprofundados, uma vez que toda análise sobre o uso dos critérios se deu apenas a partir da percepção dos gestores sem investigar a sua concretização nas empresas que somada a natureza dos estudos exploratórios de natureza qualitativa - que não intendem a realizar generalizações, constituem-se como limitações do estudo.

\section{REFERÊNCIAS}

AGOPYAN, V.; JOHN, M. V. O Desafio da Sustentabilidade na Construção Civil. São Paulo: Edgard Blucher, 2011.

BARBOSA, F.; et al. Reinventing construction: a route to higher productivity. Houston: McKinsey Global Institute, 2017.

BASSIONI, H. A.; PRICE, A. D. F.; HASSAN, T. M. Building a conceptual framework for measuring business performance in construction: an empirical evaluation. Construction Management and Economics, v. 23, n. 5, p. 495-507, 2005.

BASSIONI, H. A.; HASSAN, T. M.; PRICE, A. D. F. Evaluation and analysis of criteria and sub-criteria of a construction excellence model. Engineering, Construction and Architectural Management, v. 15, n. 1, p. 21-41, 2008.

CÂNDIDO, L. F; HEINECK, L. F. M; BARROS NETO, J. P. A Critical analysis on earned value management $(\mathrm{EVM})$ technique in building construction. In: ANNUAL CONFERENCE OF THE INTERNATIONAL GROUP FOR LEAN CONSTRUCTION, 22., 2014, Oslo.

Anais... Oslo: IGLC, 2014.

CÂNDIDO, L. F.; LIMA, S. H. O.; BARROS NETO, J. P. B. Análise de sistemas de medição de desempenho na indústria da construção. Ambiente Construído, Porto Alegre, v. 16, n. 2, p. 189-208, abr./ jun, 2016.

CHIVERALLS, K. et al. Constructing Corporate Social Responsibility: Encouraging CSR through Legislation and Regulation. RICS Construction and Property Conference. Anais... Salford and Manchester: RICS, 2011. 
SIBRAGEC - ELAGEC 2019 - de 23 a 25 de Outubro - LONDRINA - PR

CHIZZOTI, A. Pesquisa qualitativa em ciências humanas e sociais. 6. ed. Petrópolis: Vozes, 2011.

COLLIS, J.; HUSSEY, R. Pesquisa em administração: um guia prático para alunos de graduação e pós-graduação. 2. ed. Porto Alegre: Bookman, 2005.

COOPER, D. R.; SCHINDLER, P. S. Métodos de pesquisa em administração. 12. ed. Porto Alegre: AMGH, 2016.

GIBBS, G. Análise de dados qualitativos. Porto Alegre, RS: Artmed, 2009.

KOLK, A.; PINKSE, J. Stakeholder mismanagement and corporate social responisbility crisis. European Management Journal Management, v. 24, n. 1, p. 59-72, 2006.

LEONE, N. M. C. P. G. As especificidades das pequenas e médias empresas. Revista de Administração, São Paulo v.34, n.2, p91-94, abril/junho 1999.

LEONE, R. J. G.; LEONE, N. M. C. P. G. Pequenas e médias empresas: contribuições para a discussão sobre por que e como medir o seu tamanho. Revista Eletrônica do Mestrado Profissional em Administração da Universidade Potiguar, v. 4, n. 1, p. 67-83, 2011.

MACHADO FILHO, C.; ZYLBERSZTAJN, D. Responsabilidade Social Corporativa e a Criação de Valor para as Organizações. Revista de Administração da UNIMEP, v. 1, n. 1, 2003.

MAYA, R. A. Performance Management for Syrian Construction Projects. International Journal of Construction Engineering and Managament, v. 5, n. 3, p. 65-78, 2016.

MELLO, L. C. B. B.; de AMORIM, S. R. L. O subsetor de edificações da construção civil no Brasil: uma análise comparativa em relação à União Europeia e aos Estados Unidos. Produção, v. 19, n. 2, p. 388-399, 2009.

MONTEIRO FILHA, D. C.; COSTA, A. C. R. DA; ROCHA, É. R. P. DA. Perspectivas e desafios para inovar na construção civil. BNDES Setorial, v. 31, p. 353-410, 2010.

RICHARDSON, R. J. Pesquisa Social: métodos e técnicas. 3. ed. São Paulo: Atlas, 2011.

SANTINI, et al. Fatores de mortalidade em micro e pequenas empresas: um estudo na região central do rio grande do sul. Revista Eletrônica de Estratégia \& Negócios, v.8, n.1, 2015.

SOUSA, E. R. A de. Um estudo dos modelos de gestão utilizados nas pequenas empresas. 2003. 122 f. Dissertação (Mestrado em Controladoria e Contabilidade Estratégica) - Faculdade Escola de Comércio Álvares Penteado, São Paulo, 2003.

SOUSA, D. S. V. de; CÂNDIDO, L. F.; BARROS NETO, J. D. P. Medição de desempenho na construção civil - um estudo exploratório com construtoras cearenses. Ambiente Construído, v. 18, n. 1, p. 9-29, 2018.

SOUZA, et al. Pequenas empresas industriais de longa permanência no mercado: uma análise a partir da literatura e de evidências empíricas. Gestão \& Produção, São Carlos, v. 21, n. 1, p. 157-170, 2014.

TOOR, S. R..; OGUNLANA, S. O. Beyond the 'iron triangle': stakeholder perception of key performance indicators (KPIs) for large-scale public sector development projects. International Journal of Project Management. v. 28, n. 3, p. 228-236, 2010.

VILLARREAL, K. L.; PELLICER, E.; RODRIGUEZ, S. G. Performance indicators for developer and homebuilder Mexican companies: a Delphi Study. Revista de la Construccion, v. 16, n. 1, p. 133-144, 2017.

\section{AGRADECIMENTOS}

A Pró-Reitoria de Assuntos Estudantis (PRAE) da Universidade Federal do Ceará (UFC) pelo apoio recebido e aos gestores participantes da pesquisa. 